\title{
MODELLING THE ENERGY RELEASE PROCESS OF AFTERSHOCKS
}

\author{
Gospodinov D. \\ Plovdiv University 'Paisiy Hilendarski', Faculty of Physics, 4000, Plovdiv, Bulgaria, drago@uni- \\ plovdiv.bg
}

\begin{abstract}
A stochastic model for the study of Benioff strain release during aftershock sequences is suggested. The stochastic model is elaborated after a compound Poisson process and is applied on data of the M7.1 Ocober 18, 1989 Loma Prieta aftershock sequence in northern California, USA. The temporal evolution of the number of events is first modelled by the Restricted Epidemic Type Aftershock Sequence (RETAS) model and then the identified best fit model is incorporated in the energy release analysis. The suggested model is based on the assumptions that there is no relation between the magnitude and the occurrence time of an event first and second, that there is no relation between the magnitude of a certain event and magnitudes of previous events. The obtained results from the examination of the energy release reveal that the suggested model makes a good fit of the aftershock Benioff strain release and enables a more detailed study by identifying possible deviations between data and model. The real cumulative energy release values surpass the expected model ones, which proves that aftershocks, stronger than forecasted by the model, are clustered at the beginning of the Loma Prieta sequence.

Keywords: aftershock sequence, Benioff energy, stochastic model.
\end{abstract}

\section{Introduction}

The Loma Prieta M7.1 earthquake occurred on October $18^{\text {th }}$, 1989. Centered near Loma Prieta peak in the Santa Cruz Mountains south of San Jose, the quake killed 63 people and injured another 350. It caused an estimated $\$ 6$ to $\$ 10$ billion in property loss. It was the largest temblor to jolt the Bay Area since the Great San Francisco Earthquake of 1906 (magnitude 7.9). Although the Loma Prieta earthquake struck on the outskirts of the region, it exposed the vulnerability of the Bay Area to future earthquakes. This fact was reemphasized on August 24, 2014, when a magnitude 6.0 earthquake occurred near Napa, California, about 30 miles north-northeast of San Francisco, the economic losses of which are estimated to be at minimum $\$ 362$ million.

Almost certainly, some future earthquakes will certainly be larger and closer to the Bay Area's urban core than the 1989 and 2014 earthquakes. Since the Loma Prieta earthquake, many institutions have redoubled efforts to understand earthquake hazards in urban areas and to apply this new knowledge to reduce future losses. Communication of earthquake-hazard information to the public, to businesses, and to government agencies has also been strengthened.

Seismic hazard analysis studies usually do not take into account aftershock activity. This tendency is implemented by carrying out different declustering algorithms that remove aftershocks from a catalog. Recently this tendency has been replaced by the application of a number of stochastic processes for fitting the clustering behavior of a sequence. Such an approach permits making use of 
all available information in a seismic catalog and thus aftershock data can in many cases contribute in the detection of anomalous seismicity changes and provide stochastic grounds for seismic hazard analysis (Ogata et al., 2003; Drakatos, 2000).

Researchers often study the temporal or spatio-temporal relaxation patterns after a strong earthquake by elaborating adequate stochastic models of aftershock occurrences (Ogata, 1988, 1998; Ogata et al., 2003; Gospodinov and Rotondi, 2006). Much fewer papers consider the energy distribution of aftershocks, most often exploring their recurrence law. It is, however, also important to know the aftershock energy release in time. In this study we offer a stochastic process to model Benioff strain in a single sequence. The model is elaborated on the basis of a compound Poisson process based on two main assumptions: a) there is no relation between the magnitude and the occurrence time of an event; b) there is no relation between the magnitude of a certain event and magnitudes of previous events. These assumptions seem reasonable in the absence of a physical theory underlying the relaxation process. We implemented the presented stochastic process to model Benioff strain release of the M7.1 Ocober 18, 1989 Loma Prieta aftershock sequence in northern California, USA. Comparison between data and model allows identifying and interpreting eventual deviations thus making possible to explore the aftershock process in more detail.

\section{Methodology}

\subsection{Energy release stochastic model}

For the development of a Benioff strain release model we apply an analog of a counting process fo $\mathrm{r}$ marked point processes, which is sometimes termed as a mark accumulator process and is defined as follows: let's have a Poisson process $\{N(t): t \geq 0\}$ with a rate $\lambda>0$ and suppose that the time $T_{i}$ of each event is associated to a realization of a random variable $Y_{i}$, where $\left\{Y_{n}: n>0\right\}$ is a family of $\mathrm{i}$ ndependent and identically distributed random variables sharing the distribution $G(y)=\operatorname{Pr}\left\{Y_{k} \leq y\right\}$ . One more requirement is that they have also to be independent of $\{N(t): t \geq 0\}$, and then the stocha stic process

\section{Equation 1 - compound Poisson process}

$$
\mathrm{Z}(t)=\sum_{k=1}^{N(t)} \mathrm{Y}_{k} \quad \text { for } \quad t \geq 0
$$

is said to be a compound Poisson process (Taylor and Karlin, 1984).

If $\mu$ and $v^{2}$ are the common mean and variance for the marks $Y_{1}, Y_{2}, \ldots$ then the moments of $Z(t)$ (mean and variance) are given by:

\section{Equation 2 - mean of a compound Poisson process}

$$
\mathrm{E}[Z(t)]=\lambda \mu t
$$

\section{Equation 3 - variance of a compound Poisson process}

$$
\operatorname{Var}[Z(t)]=\lambda\left(v^{2}+\mu^{2}\right) t
$$

Considering the assumptions made for the elaboration of the compound Poisson process, we see th at they coincide with the ones made earlier for the aftershocks' magnitudes. Thus this stochastic pr ocess could be used as a model process of random behavior in case a sequence of occurrence times and size of events is to be analyzed.

In the most general treatment of marked point processes $\{N(t): t \geq 0\}$ is an inhomogeneous Poisson process with an intensity function $\lambda=\lambda(t): t \geq 0$ and neither the marks $\left\{Y_{n}: n>0\right\}$ need to for $\mathrm{m}$ an independent sequence of random variables. Nor it is required for the marks to be independent of the counting process or the occurrence time sequence (Snyder and Miller, 1991). 
If we restrict ourselves to consider an inhomogeneous compound Poisson process with a rate $\lambda=\lambda($ t) then formulae (2) and (3) would be translated into:

Equation 4 - mean of an inhomogeneous compound Poisson process

$\mathrm{E}[Z(t)]=\mu \int_{t_{0}}^{t} \lambda(s) d s$

Equation 5 - variance of an inhomogeneous compound Poisson process

$\operatorname{Var}[Z(t)]=\left(v^{2}+\mu^{2}\right) \int_{t_{0}}^{t} \lambda(s) d s$

following Snyder and Miller (1991). The common mean, provided by formula (4), models the cum ulative Benioff strain release and the square root of the variance provides the error bounds.

Formulae (4) and (5) reveal that the intensity function $\lambda=\lambda(t)$ is needed in order to employ the Ben ioff strain release model. We evaluate the intensity function parameters by modelling it by the RET AS model.

\subsection{Restricted Epidemic Type Aftershock Sequence (RETAS) model}

The aftershock intensity decay function is most widely analyzed starting with the Modified Omori Formula (MOF) which follows the idea that the aftershocks are conditionally independent and follo w a nonstationary Poisson process (Utsu, 1970). Multiple strong events and complex aftershock se quences led Ogata (1988) to the formulation of the Epidemic Type Aftershock-Sequence (ETAS) by which each aftershock can trigger its own secondary events.

Gospodinov and Rotondi (2006) proposed the RETAS model, which is based on the assumption th at only aftershocks with magnitudes larger than or equal to a threshold $M_{t h}$ can induce secondary se ismicity. It has the advantage that it includes the MOF and the ETAS models as limit cases. The co nditional intensity function for this model is:

Equation 6 - conditional intensity function of the RETAS model

$$
\lambda\left(t \mid H_{t}\right)=\mu+\sum_{\substack{t_{i}<t \\ M_{i} \geq M_{t h}}} \frac{K_{0} e^{\alpha\left(M_{i}-M_{0}\right)}}{\left(t-t_{i}+c\right)^{p}}
$$

In this equation $\mu$ is the background seismicity rate, ${ }^{t}{ }_{i}$ is the occurrence time of the ith event, $p$ is a coefficient of attenuation, $c$ and $K_{o}$ are constants and $\alpha$ measures the effect of magnitude in the production of 'descendants'. $H_{t}$ is the history of the process, including all times and magnitudes of events, occurred before time $\mathrm{t}$ with magnitudes $M_{i} \geq M_{\text {th }}$. The summation in equation (6) occurs over all events with occurrence times $t_{i}<t$ and magnitudes $\mathrm{Mi} \geq \mathrm{Mth}$.

Having parameterized the conditional intensity function, a Fortran program was applied to calculat e the maximum log-likelihood function

\section{Equation 7 - log-likelihood function of the conditional intensity function}

$$
\log L(\theta)=\sum_{i=1}^{N} \log \lambda_{\theta}\left(t_{i} \mid H_{t_{i}}\right)-\int_{S}^{T} \lambda_{\theta}\left(t \mid H_{t}\right) d t
$$


and to obtain the maximum likelihood estimates (MLE) of the model parameters. Here the symbol $\theta$ denotes the model parameters $K_{0}, \mu, \quad \alpha, \quad c$ and $p, \quad \lambda_{\theta}\left(t \mid H_{t}\right)$ is the conditional intensity function of the RETAS model and $H_{t}$ is as defined for Formula 6. As magnitude values are quite discrete, $M_{t h}$ was not included as a para meter for estimation in the model. However, obtaining the MLE for different $M_{t h}$, we may chose th is value of $M_{t h}$ for which we get minimum Akaike Information Criterion (AIC), which identifies th e best fit RETAS model (Akaike, 1974).

\section{Equation 8 - Akaike Information Criterion (AIC)}

$$
A I C=-2 \max \log L+2 k
$$

Here $L$ is the likelihood function and $k$ is the number of model parameters.

\subsection{Benioff strain}

For the analyzed Loma Prieta aftershock sequence the intensity was assessed by the method of Gos podinov et al. (2007) through the RETAS model and the estimated parameters are presented in Tab le 1 . In the present paper we use the latter results to model the seismic release rate in an aftershock sequence. We have chosen Benioff strain, $\varepsilon$, as a measure of seismic energy release, which is the sq uare root of the seismic energy, and then the cumulative Benioff strain is $\varepsilon(t)=\sum_{i=1}^{N} \varepsilon_{i}=\sum_{i=1}^{N} \sqrt{E_{i}(t)}$. This measure is particularly useful when smaller events are also con sidered as in the current case (Tzanis and Vallianatos, 2003). In the above formula $E_{i}(t)$ is the en ergy of the $i^{\text {th }}$ event and $N$ is the total number of events at time $t$.

\section{Data and results}

There is a huge amount of data, compiled for the relaxation process after the Loma Prieta earthquake. In 1991, the Loma Prieta Data Archive was established with funds from the US Geological Survey (USGS) to gather, organize, and archive raw data associated with the Loma Prieta earthquake.

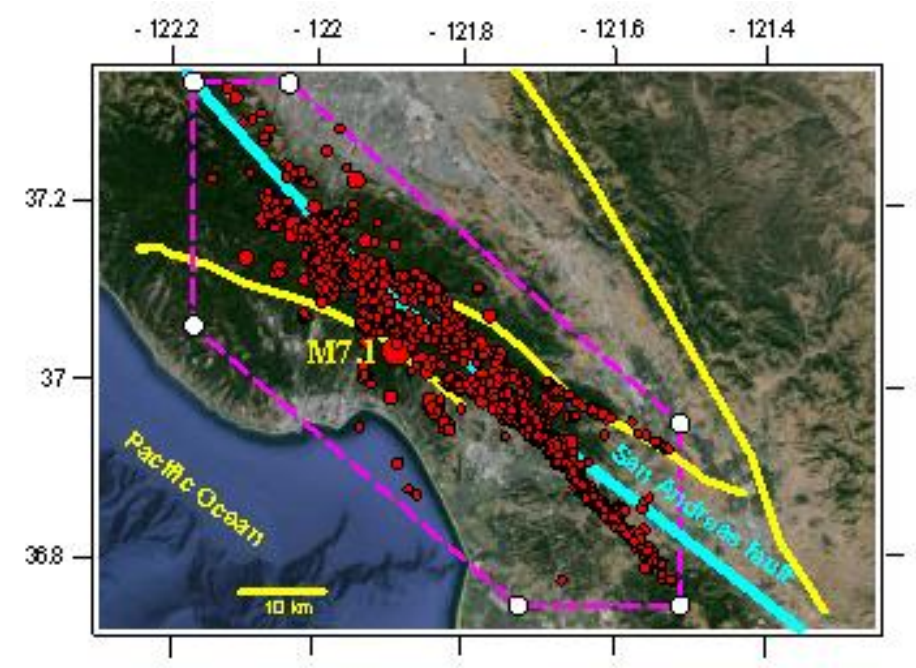

Figure 1 - Epicentral map of the 1989 M7.1 Loma Prieta earthquake. Circles denote epicentres, the thick line stands for the San Andreas fault and the thinner lines reveal nearby faults. Dashed line delineates the polygon, covering the locations of the examined events. 
The purpose was to complement the National Earthquake Hazards Program (NEHRP) issued official report to Congress describing the post-earthquake investigation of the October 17, 1989, Loma Prieta, California earthquake. Twenty-four data sets are included in the archive. All were contributed by the researchers on a voluntary basis. The datasets address strong motion, aftershocks, geotechnical, structural and social survey data. In 1991, nonprint data were seldom published in the open literature so it was useful to try to organize and preserve the data for future scholarship.

The file 1p8910.zip from the Loma Prieta Data Archive (Dietz and Ellsworth, 1991) includes all recorded earthquakes (10373 events) from Oct 18, 1989 to Oct 17, 1991 which locate in the polygon defined by these corners:

$\begin{array}{cc}\text { Lat } & \text { Long } \\ 37.33 & -122.17 \\ 37.33 & -122.03 \\ 36.95 & -121.49 \\ 36.75 & -121.49 \\ 36.75 & -121.72 \\ 37.06 & -122.17 \\ 37.33 & -122.17\end{array}$

All suspected quarry blasts have been removed from this listing. A detailed look at the early aftershock catalog shows a completeness level $\sim$ M1.5 after hour 6 of the aftershock sequence. We analyzed data of the Loma Prieta aftershock sequence, containing N=1024 events with magnitude $\mathrm{M} \geq 2.0$ for the first year after the strong earthquake.

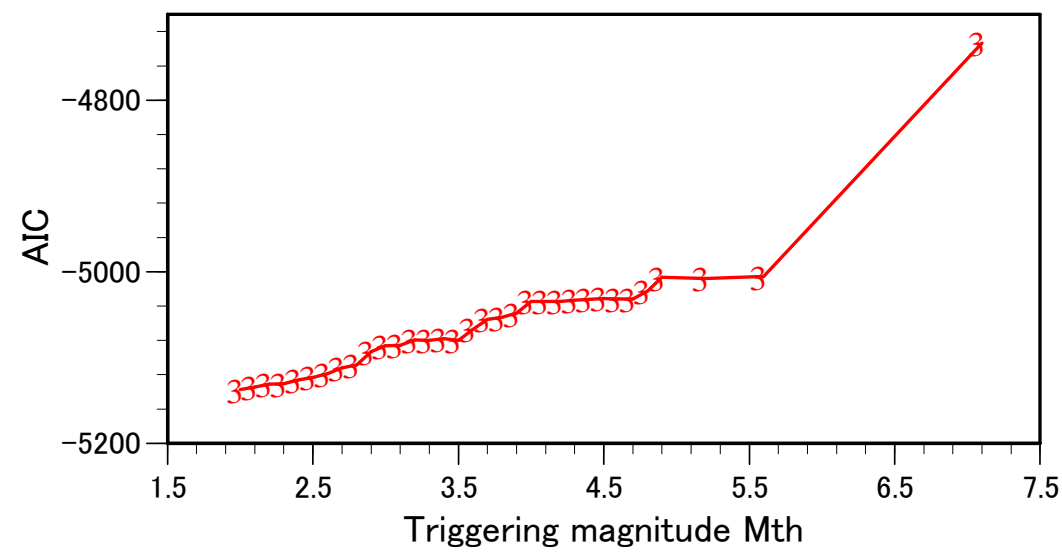

Figure 2 - The AIC values depending on the triggering magnitude values. The minimum value of $\mathrm{AIC}$ is when $\mathrm{M}_{\mathrm{th}}$ equals the lowest cutoff magnitude of the sample, which reveals that the best fit RETAS version is when it coincides with ETAS.

We first applied the RETAS model to fit the temporal decay of aftershocks rate decay in time. The lowest value of AIC is for $\mathrm{M}_{\mathrm{th}}$ equal to the lowest cutoff magnitude of the sample. Thus, the identified best fit RETAS version concides with the ETAS model. The MLEs of the best fit model parameters are presented in Table 1. The recognized clustering pattern discloses that events are grouped temporally not only to stronger aftershock, but also to weaker ones.

Table 1 - RETAS model parameters (see formula (6)) of the best fit model.

\begin{tabular}{|c|c|c|c|c|c|c|c|}
\hline Model & $\mathbf{M}_{\text {th }}$ & AIC & $\boldsymbol{\mu}$ & $\mathbf{K}$ & $\boldsymbol{\alpha}$ & $\mathbf{c}$ & $\mathbf{p}$ \\
\hline ETAS (best) & 2 & -5137.16 & 0 & 0.015 & 1.511 & 0.019 & 1.12 \\
\hline
\end{tabular}


The recognized best fit model provided the necessary conditional intensity $\lambda_{\theta}\left(t \mid H_{t}\right)$, which was later incorporated in Formulae 4 and 5 to model Benioff energy release in the sequence. The results for the obtained best fit temporal model (ETAS) and for the energy release were plotted on Figure 3.

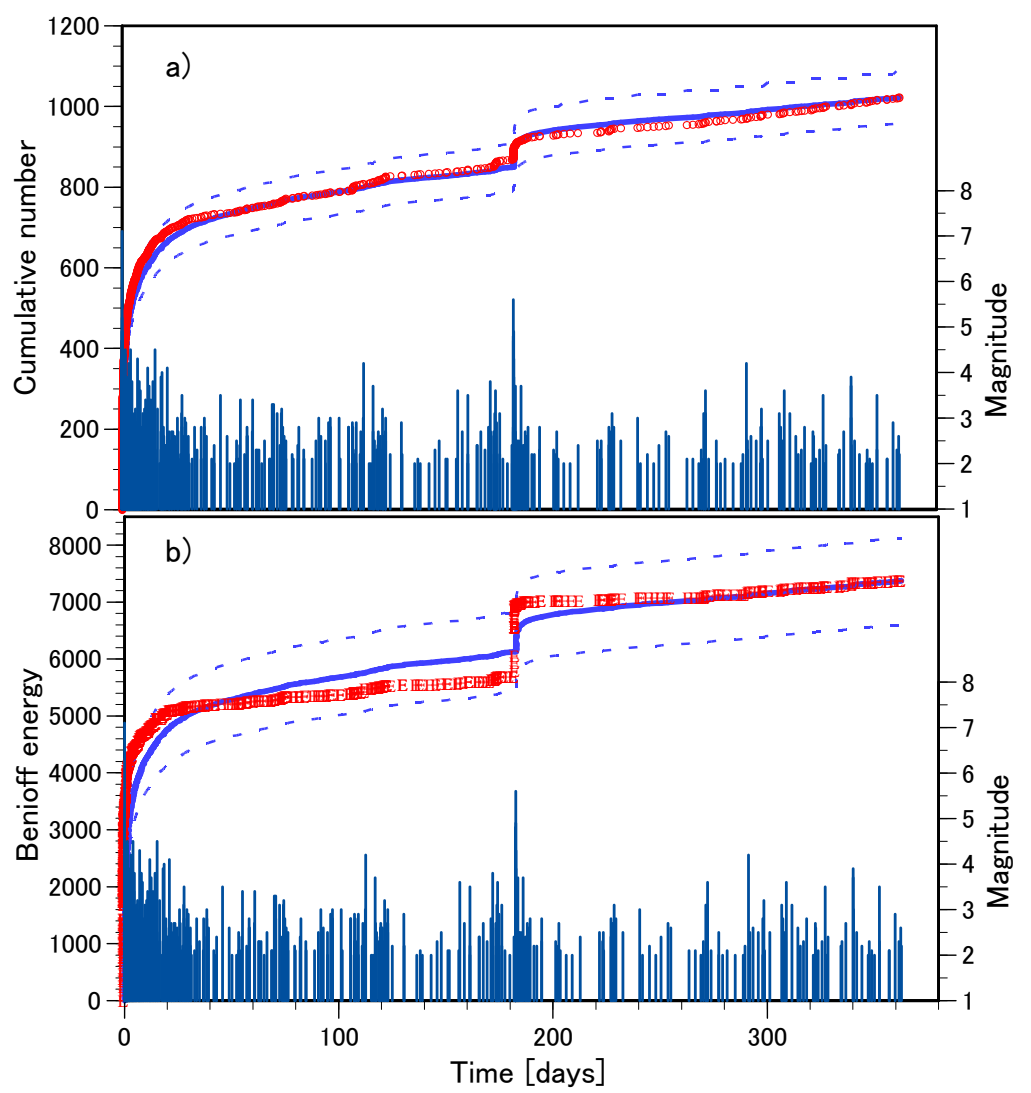

Figure 3 - Cumulative number and Benioff energy evolution with time for one year period:

a) Cumulative number - circles stand for the real cumulative number, the thick line represents expected cumulative number and dashed lines are the error bounds. Vertical lines stand for events' magnitudes; b) Benioff energy - circles denote real energy released, thick line reveals the expected released energy values, calculated after Formula 4 and the dashed lines are the error bounds, presented by the standard deviation.

We examined how well the ETAS model fits real data by comparing real cumulative number of events in time with the one calculated by the MLEs. Standard deviation was used for an error estimate and one can see both curves on Figure 3a. The circles stand for the real cumulative number, the thick line represents expected cumulative number and dashed lines are the error bounds.

Generally, it can be seen that the model fits real data quite well. Although there is some exceedance of real over model cumulative number at the beginning of the sequence, both curves stay within the error bounds for the entire one year period.

On Figure 3b we have shown Benioff energy evolution with time during the aftershock sequence. Here, again circles denote real energy released, thick line reveals the expected released energy values, calculated after Formula 4 and the dashed lines are the error bounds, presented by the standard deviation (see Formula 5).

Here some stronger divergences between model and real values are to be seen. The time scale (one year), however, does not allow capturing details of the observed divergence at the beginning of the 
sequence. That is why we performed another contrast of real and model Benioff energy release, this time only for a period of five days. The results are plotted on Figure 4.

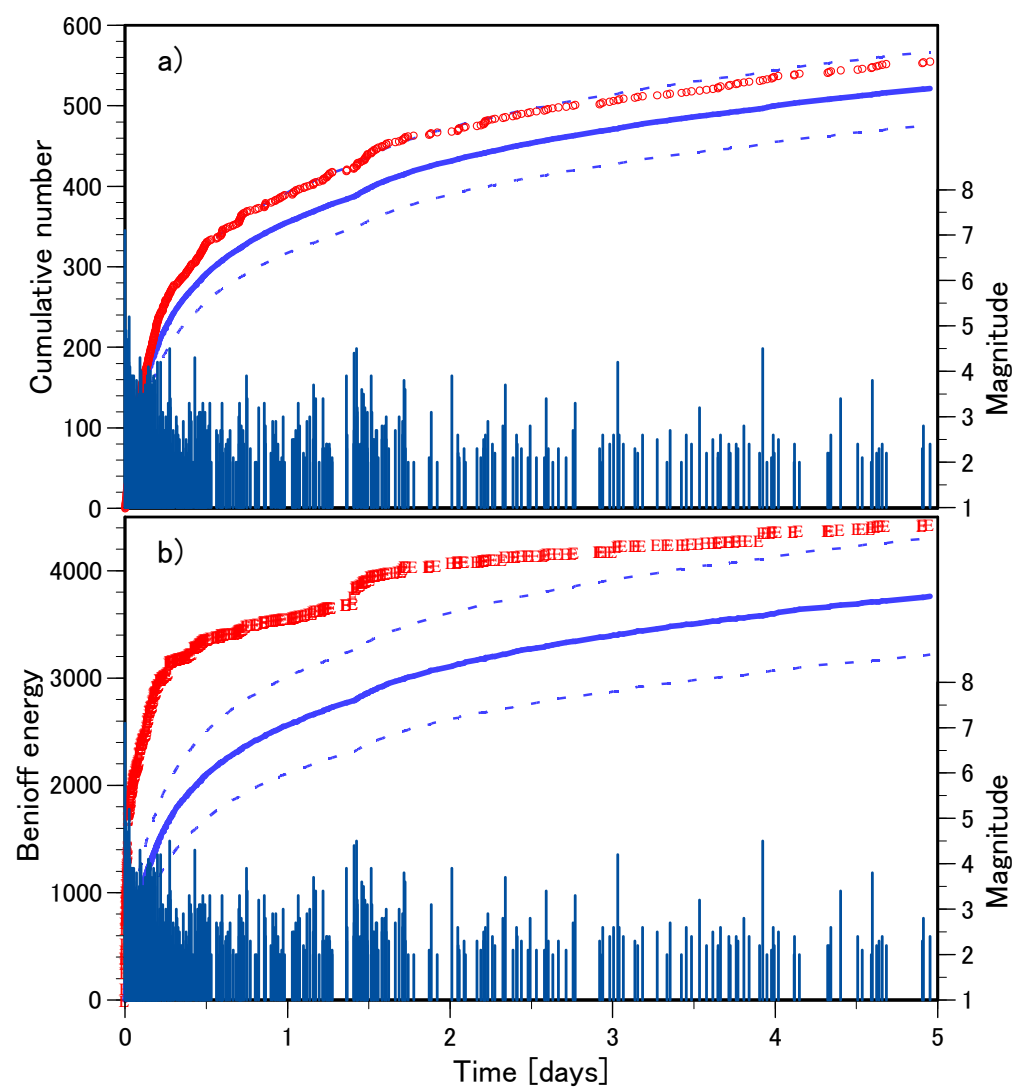

Figure 4 - Cumulative number and Benioff energy evolution with time for five days period Notation as in Figure 3.

We can see on Figure 4a that real and expected cumulative values remain within the error bounds although there is a small discrepancy between them. The picture is quite different for the energy release, exposed on Figure 4b. The difference there between model and real values is significant, real rate of energy release exceeding substantially the model rate. This is especially true for the first 6 hours of the first day, proving that we have a cluster of stronger aftershocks at the beginning, not forecasted by the model.

\section{Discussion}

In this paper we offered a stochastic model for patterning the Benioff strain release during the Loma Prieta aftershock sequence. We first examined the temporal evolution of the number of events by the RETAS model and then the identified best fit model was incorporated in the energy release analysis. While the temporal model follows very closely the actual data, a certain discrepancy is observed between model and data for the energy distribution. Benioff strain release for the sequence exceeds the model for the first several days, which is a clear indication of increased aftershock clustering for the beginning of the sequence. The grouping is stronger than suggested by the model for independent events. 
The results obtained demonstrate that the operated stochastic model enables identification of specific features of the Benioff energy evolution in time. This stochastic pattern can be used as a benchmark model, developed after suppositions of seismic events independence in the sequence.

\section{Acknowledgments}

This research was financed by the University of Plovdiv "Paisii Hilendarski”, under contract № NI15-FFIT-005/23.04.2015.

\section{References}

Akaike, H., 1974. A new look at the statistical model identification, IEEE Trans Autom Control AC, 19, 716-723.

Bhattacharya, P., Kamal and Chakrabarti, B., 2009. The time distribution of aftershock magnitudes, fault geometry and aftershock prediction, arXiv: 0910.3738 [physics.geo-ph].

Dietz, L. and Ellsworth, W., 1991. LOMA PRIETA DATA ARCHIVE, National Information Service for Earthquake Engineering (NISEE), http://nisee.berkeley.edu/lp_archive/aftershk.html.

Drakatos, G., 2000. Relative seismic quiescence before large aftershocks, Pure Appl. Geophys. 157, 1407-1421.

Gospodinov, D. and Rotondi, R., 2006. Statistical analysis of triggered seismicity in the Kresna Region of SW Bulgaria (1904) and the Umbria-Marche Region of Central Italy (1997), Pure Appl. Geophys. 163, 1597-1615.

Gospodinov D., Papadimitriou E., Karakostas, V. and Ranguelov, B., 2007. Analysis of relaxation temporal patterns in Greece through the RETAS model approach, Phys. Earth Planet. Inter., 165/3-4, 158-175, doi: 10.1016/j.pepi.2007.09.001.

Ogata, Y., 1988. Statistical models for earthquake occurrences and residual analysis for point processes, J. Am. Stat. Assoc., 83, 9-27.

Ogata, Y., 1998. Space-time point-process models for earthquake occurrences, Ann. Inst. Stat. Math., 50, 379-402.

Ogata, Y., Jones, L.M. and Toda, S., 2003. When and where the aftershock activity was depressed: contrasting decay patterns of the proximate large earthquakes in southern California, $J$. Geophys. Res., 108, 2318, doi: 10.1029/2002JB002009.

Snyder, D. and Miller, M., 1991. Random point processes in time and space, Springer-Verlag, 481 pp.

Taylor, H. and Karlin, S., 1984. An introduction to stochastic modeling: Academic Press, Inc., 399 pp.

Tzanis, A. and Vallianatos, F., 2003. Distributed power-law seismicity changes and crustal deformation in the SW Hellenic arc, Natural Hazards and Earth System Sciences, 3, 179-198.

Utsu, T., 1970. Aftershocks and earthquake statistics (II): further investigation of aftershocks and other earthquake sequences based on a new classification of earthquake sequences, J. Fac. Sci., Hokkaido Univ., Ser. VII (Geophys.) 3, 198-266. 